\title{
Evaluation on Mean Performance in Bottle Gourd [Lagenaria siceraria (Molina) Standl] Genotypes
}

\author{
Akshay Jain*, S.P. Singh, Rajneesh Shukla and Sriom \\ Department of Vegetable Science Narendra Deva University of Agriculture and Technology \\ Kumarganj, Faizabad-224229(U.P.), India \\ *Corresponding author
}

K e y w o r d s
Mean performance,
$\begin{aligned} & \text { Genotypes, Fruit } \\ & \text { yield, Bottle gourd }\end{aligned}$
Article Info
$\begin{aligned} & \text { Accepted: } \\ & \text { 20 March } 2018 \\ & \text { Available Online: } \\ & \text { 10 April } 2018\end{aligned}$

\section{Introduction}

Bottle gourd [Lagenaria siceraria (Molina) Standl, 2n $=22$ ] also called as birdhouse gourd, trumpet gourd, white flowered gourd and calabash gourd, is one of the most ancient crops with its man's association since 12000 B.C., as indicated by archeological remains in Peru (Esquinas-Al cazae and Gulick, 1983). It is a popular cucurbitaceous crop in India and cultivated in other tropical and sub-tropical regions of the world. The tender fruits are also used to prepare sweets, rayata and pickles. The dried shells of mature fruits are extremely hard and are used as containers, utensils, musical instruments, floats of fishnets or ornamental items. The leaves are also used to prepare vegetable and they have higher nutritive value than fruits, in respect of protein, fat, minerals, fibre, carbohydrate, energy, calcium and phosphorus contents (Gopalan et al., 1982). Different plant parts of bottle gourd have several putative medicinal properties (Chopra, 1986; Moreman, 1998; Chaudhary, 2001; Manandhar, 2002).

As per FAO, estimates the world acreage under gourd and squashes is about 1.462 million hectares with a total annual production of 18.98 million tonnes with the productivity 
of 12.97 tonnes per hectare (Anonymous, 2004). Bottle gourd occupies a prominent position among various cucurbit crops grown in India. It is cultivated in an area of 1.17 lakh hectares with a production of 1.42 million tonnes leading to a productivity of 12.12 tonnes per hectare (Sidhu, 2002). Like other cucurbits - bottle gourd is a summer season vegetable under the north Indian climatic conditions, it is mainly cultivated both in spring- summer (February to June) and rainy (July to November) seasons.

\section{Materials and Methods}

The experimental material comprised of forty genotypes of bottle gourd with two checks was evaluated at Main Experiment Station of Department of Vegetable Science, at Narendra Deva University of Agriculture and Technology, Narendra Nagar (Kumarganj), Faizabad (U.P.). Geographically the experimental site falls under humid subtropical climate and is located in between $24.47^{\circ}$ and $26.56^{\circ} \mathrm{N}$ latitude, and $82.12^{\circ}$ and $83.58^{\circ}$ E longitude at an altitude of $113 \mathrm{~m}$ above the mean sea level in the Gangetic Alluvial Plains of eastern Uttar Pradesh.

The experiment was conducted in Randomized Block Design with three replications to evaluate forty bottle gourd genotypes. Individual plot dimension was $3 \mathrm{~m}$ $\mathrm{x} 3 \mathrm{~m}$ with a row to row spacing of $3 \mathrm{~m}$ and plant to plant spacing of $50 \mathrm{~cm}$. Thus six plants were maintained in individual plots. Recommended doses of F.Y.M. and fertilizers were applied. The required pesticides and fungicides were used, as and when required to save the crop from pests and diseases, respectively. The observations were recorded from five randomly selected plants from each treatment in each replication. Observations on the following parameters were recorded using the standard procedure: node number to first staminate flower anthesis, node number to first pistillate flower anthesis, days to first staminate flower anthesis, days to first pistillate flower anthesis, days to first harvest, fruit length/polar length $(\mathrm{cm})$, fruit circumference $(\mathrm{cm})$, fruit weight $(\mathrm{kg})$, number of fruits/plant, vine length at the time of last harvest $(\mathrm{m})$, number of primary branches/vine at the time of last harvest, fruit yield (kg/plant) and fruit yield ( $\mathrm{q} / \mathrm{ha})$. The replicated mean data recorded will be analyzed according to Panse and Sukhatme (1989). Coefficient of variability is according to Burton and de Vane (1953). Heritability as suggested by Hanson et al., (1956). Genetic advanced (GA) was calculated by the method suggested by Johnson et al., (1955), and Correlation coefficient as suggested by Al-Jibouri et al., (1958). The Experimental was laid out in an RBD design and data were calculated with analysis of variance (ANOVA)

\section{Results and Discussion}

The mean performance for adjusted mean, range and least significant difference of 40 bottle gourd genotypes for 13 characters are presented in table node number to first staminate flower anthesis varied from NDMLNS-11 (5.30) to General mean of trait for all genotypes ware found (7.38). Node number to first pistillate flower anthesis varied from NDML-SS-15 (6.20) to General mean of this trait for all genotypes was (10.88) days to first staminate flower anthesis NDML-NS-11 (42.67 days) to General mean of this trait for all genotypes was (46.97). days to first pistillate flower anthesis NDML-SS-17 in (44 days) to General mean of this trait for all genotypes was (49.11) days to first harvest NDML-SS-13 (54.00) to 63.33 General mean of this trait for all genotypes was (58.77 days), fruit length/polar length $(\mathrm{cm})$ NDML-SS-13 (18.63) to NDML-SS-16 (24.37) General mean of this trait for all genotypes was (30.29) fruit circumference (cm) NDBG-619-6-4 (19.60) to NDML-SS-18 (43.17) 
Table.1 Mean performance of the forty bottle gourd genotypes for 13 economic traits

\begin{tabular}{|c|c|c|c|c|c|c|c|c|c|c|c|c|c|c|}
\hline \multirow[t]{2}{*}{ SN } & \multirow[t]{2}{*}{ Characters } & \multirow[t]{2}{*}{$\begin{array}{l}\text { Node } \\
\text { number to } \\
\mathbf{1}^{\text {st }} \\
\text { staminate } \\
\text { flower } \\
\text { anthesis } \\
\mathbf{1}\end{array}$} & \multirow[t]{2}{*}{$\begin{array}{l}\text { Node } \\
\text { number } \\
\text { to } 1^{\text {st }} \\
\text { pistillate } \\
\text { flower } \\
\text { anthesis } \\
2\end{array}$} & \multirow[t]{2}{*}{$\begin{array}{l}\text { Days to } \\
1^{\text {s }} \\
\text { stamina } \\
\text { te } \\
\text { flower } \\
\text { anthesis } \\
\mathbf{3}\end{array}$} & \multirow[t]{2}{*}{$\begin{array}{l}\text { Days to } 1^{\text {st }} \\
\text { pistillate } \\
\text { flower } \\
\text { anthesis } \\
4\end{array}$} & \multirow[t]{2}{*}{$\begin{array}{l}\text { Days to } \\
\mathbf{1}^{\text {st }} \\
\text { harvest }\end{array}$} & \multirow[t]{2}{*}{$\begin{array}{l}\text { Fruit } \\
\text { length/ } \\
\text { polar } \\
\text { length }\end{array}$} & $\begin{array}{l}\text { Fruit } \\
\text { circumf } \\
\text { erence } \\
\text { (cm) }\end{array}$ & \multirow[t]{2}{*}{$\begin{array}{l}\text { Fruit } \\
\text { weight }\end{array}$} & \multirow[t]{2}{*}{\begin{tabular}{|l} 
Numbe \\
$\mathbf{r}$ of \\
fruits/ \\
plant
\end{tabular}} & \multirow[t]{2}{*}{$\begin{array}{l}\text { Vine } \\
\text { length } \\
(\mathrm{m})\end{array}$} & \multirow[t]{2}{*}{$\begin{array}{l}\text { Primary } \\
\text { branches/ } \\
\text { vine at } \\
\text { last } \\
\text { harvelst }\end{array}$} & \multirow[t]{2}{*}{\begin{tabular}{|l}
$\begin{array}{l}\text { Fruit } \\
\text { yield } \\
\text { (kg } \\
\text { /plant) }\end{array}$ \\
$\mathbf{1 2}$ \\
\end{tabular}} & $\begin{array}{l}\text { Fruit yield } \\
(\mathbf{q} / \mathbf{h a})\end{array}$ \\
\hline & & & & & & & & 7 & & & & & & 13 \\
\hline \multicolumn{15}{|c|}{ Long fruited pale green genotypes } \\
\hline 1 & NDBG-140 & 6.13 & 9.53 & 45.00 & 48.00 & 56.33 & 34.70 & 23.57 & 0.89 & 5.10 & 6.73 & 14.67 & 5.01 & 303.96 \\
\hline 2 & NDBG-601-1 & 7.77 & 12.30 & 48.33 & 51.00 & 60.33 & 40.93 & 20.80 & 0.96 & 4.87 & 6.03 & 13.08 & 4.54 & 302.64 \\
\hline 3 & NDBG-601-2 & 8.00 & 12.00 & 49.00 & 50.67 & 60.00 & 46.27 & 21.90 & 0.94 & 6.03 & 5.59 & 14.17 & 5.53 & 368.64 \\
\hline 4 & NDBG-601-3 & 6.50 & 11.30 & 45.00 & 50.33 & 60.67 & 49.33 & 21.97 & 0.97 & 5.53 & 7.30 & 15.33 & 5.14 & 342.60 \\
\hline 5 & NDBG-601-4 & 6.40 & 11.93 & 45.00 & 50.00 & 59.00 & 46.43 & 20.87 & 0.95 & 4.37 & 5.16 & 13.00 & 4.24 & 282.66 \\
\hline 6 & NDBG-619-6-1 & 7.60 & 11.80 & 45.00 & 48.00 & 59.33 & 43.57 & 22.37 & 0.85 & 4.83 & 5.09 & 13.39 & 4.23 & 281.94 \\
\hline 7 & NDBG-619-6-2 & 6.70 & 10.37 & 45.67 & 48.67 & 60.00 & 46.49 & 21.33 & 0.91 & 3.37 & 4.90 & 10.45 & 3.12 & 207.96 \\
\hline 8 & NDBG-619-6-3 & 8.20 & 11.53 & 48.67 & 48.33 & 58.67 & 41.67 & 21.23 & 0.82 & 4.53 & 5.58 & 15.32 & 3.71 & 247.26 \\
\hline 9 & NDBG-619-6-4 & 9.50 & 13.83 & 49.67 & 51.67 & 59.33 & 46.63 & 19.60 & 0.93 & 5.37 & 5.50 & 12.67 & 5.19 & 345.96 \\
\hline 10 & NDBG-619-11-1 & 7.53 & 10.33 & 45.67 & 47.33 & 55.67 & 41.23 & 22.87 & 0.94 & 5.90 & 4.66 & 10.08 & 5.28 & 351.96 \\
\hline 11 & NDBG-619-11-2 & 8.80 & 11.23 & 46.67 & 45.33 & 57.67 & 42.33 & 21.50 & 0.95 & 8.37 & 6.24 & 11.67 & 7.95 & 529.92 \\
\hline 12 & NDBG-619-11-3 & 8.73 & 12.63 & 46.67 & 47.00 & 60.00 & 41.23 & 21.47 & 0.93 & 6.03 & 7.15 & 14.33 & 5.60 & 373.32 \\
\hline 13 & NDBG-701 & 8.57 & 11.43 & 46.00 & 51.33 & 59.33 & 42.00 & 21.30 & 0.94 & 6.77 & 5.68 & 11.53 & 6.22 & 414.60 \\
\hline 14 & NDBG-703 & 8.27 & 10.13 & 47.67 & 51.00 & 60.00 & 36.60 & 21.93 & 0.84 & 6.90 & 4.62 & 10.67 & 6.22 & 414.60 \\
\hline 15 & $\begin{array}{l}\text { Narendra Rashmi } \\
\text { (Check) }\end{array}$ & 7.30 & 11.80 & 45.67 & 47.33 & 60.33 & 36.43 & 23.17 & 0.87 & 6.27 & 6.53 & 12.50 & 5.95 & 396.60 \\
\hline \multicolumn{15}{|c|}{ Long fruited striped green genotypes } \\
\hline 16 & NDBG-613-1 & 10.37 & 15.60 & 50.23 & 52.00 & 62.67 & 35.37 & 22.57 & 0.79 & 4.37 & 5.93 & 12.12 & 3 & 230.64 \\
\hline 17 & NDBG-613-2 & 8.00 & 15.13 & 49.33 & 51.67 & 63.33 & 37.10 & 23.07 & 0.74 & 1.97 & 5.29 & 12.58 & 1.46 & 97.32 \\
\hline 18 & NDBG-613-3 & 7.20 & 13.23 & 45.00 & 47.67 & 56.33 & 42.37 & 23.00 & 0.90 & 3.50 & 6.17 & 14.77 & 3.31 & 220.62 \\
\hline 19 & $\begin{array}{l}\text { Narendra } \\
\text { Dharidar (Check) }\end{array}$ & 9.43 & 11.23 & 48.33 & 50.00 & 60.00 & 34.77 & 22.97 & 0.87 & 8.50 & 6.04 & 15.25 & 7.83 & 521.94 \\
\hline Rou & Id fruited pale gree & deter & & & & & & & & & & & & \\
\hline 20 & RDPG-NS-1 & 6.97 & 9.63 & 47.00 & 51.00 & 59.00 & 18.97 & 41.73 & 0.89 & 2.67 & 6.03 & 10.10 & 2.71 & 180.66 \\
\hline 21 & RDPG-NS-2 & 5.87 & 8.93 & 45.00 & 50.33 & 58.00 & 22.57 & 33.88 & 0.84 & 2.8 & 5.08 & 12.00 & 2.96 & 197.28 \\
\hline 22 & RDPG-NS-3 & 6.03 & 9.47 & 46.33 & 48.67 & 57.67 & 20.93 & 36.33 & 0.84 & 4.80 & 4.28 & 6.66 & 3.99 & 265.92 \\
\hline Rou & d fruited striped g & een genotyp & & & & & & & & & & & & \\
\hline 23 & NDML-SS-1 & 7.17 & 11.40 & 47.00 & 51.87 & 61.33 & 19.00 & 39.92 & 0.89 & 5.63 & 5.15 & 11.81 & 5.19 & 345.96 \\
\hline 24 & NDML-SS-2 & 8.03 & 11.87 & 48.67 & 50.00 & 60.33 & 18.87 & 39.30 & 0.92 & 3.03 & 5.25 & 11.02 & 2.75 & 183.30 \\
\hline 25 & NDML-SS-3 & 6.93 & 10.70 & 47.00 & 49.00 & 60.00 & 19.42 & 42.83 & 0.91 & 3.13 & 6.58 & 11.00 & 3.41 & 227.28 \\
\hline 26 & NDML-SS-4 & 7.17 & 8.47 & 48.67 & 48.00 & 60.00 & 19.20 & 40.77 & 0.92 & 3.80 & 6.17 & 9.42 & 3.66 & 243.96 \\
\hline 27 & NDML-SS-5 & 6.83 & 10.30 & 49.60 & 52.33 & 58.67 & 19.77 & 39.73 & 0.87 & 5.37 & 6.97 & 13.33 & 4.53 & 301.98 \\
\hline 28 & NDML-SS-9 & 6.27 & 7.50 & 47.67 & 47.67 & 59.67 & 19.27 & 40.83 & 0.98 & 5.83 & 6.51 & 14.22 & 6.66 & 444.00 \\
\hline 29 & NDML-SS-13 & 5.60 & 7.80 & 45.67 & 46.33 & 54.00 & 18.63 & 37.80 & 0.91 & 3.93 & 4.51 & 7.33 & 3.45 & 229.98 \\
\hline 30 & NDML-SS-14 & 6.93 & 7.07 & 46.00 & 46.33 & 56.67 & 21.33 & 39.50 & 0.85 & 2.53 & 4.84 & 13.27 & 2.12 & 141.30 \\
\hline 31 & NDML-SS-15 & 5.57 & 6.20 & 45.00 & 44.33 & 54.00 & 20.13 & 38.50 & 0.86 & 2.60 & 3.84 & 7.42 & 2.25 & 150.00 \\
\hline 32 & NDML-SS-16 & 8.33 & 10.80 & 48.00 & 51.00 & 59.67 & 24.37 & 34.50 & 0.91 & 4.30 & 4.40 & 8.33 & 3.73 & 248.64 \\
\hline 33 & NDML-SS-17 & 7.80 & 8.30 & 45.00 & 44.00 & 54.67 & 22.53 & 40.30 & 0.97 & 1.60 & 5.06 & 7.78 & 1.54 & 102.66 \\
\hline 34 & NDML-SS-18 & 5.77 & 7.50 & 46.67 & 50.00 & 59.00 & 19.08 & 43.17 & 1.03 & 1.97 & 6.65 & 11.56 & 2.01 & 133.98 \\
\hline 35 & NDML-NS-6 & 6.93 & 10.90 & 48.33 & 49.33 & 60.33 & 19.73 & 40.47 & 0.91 & 6.67 & 6.26 & 12.58 & 5.78 & 385.26 \\
\hline 36 & NDML-NS-7 & 6.40 & 8.93 & 45.30 & 48.67 & 54.67 & 19.43 & 38.70 & 0.94 & 4.93 & 5.69 & 12.00 & 4.75 & 316.62 \\
\hline 37 & NDML-NS-8 & 7.93 & 11.37 & 46.67 & 49.33 & 58.67 & 21.20 & 37.13 & 0.91 & 4.27 & 4.72 & 13.67 & 3.94 & 262.62 \\
\hline 38 & NDML-NS-10 & 8.53 & 12.13 & 49.33 & 51.33 & 61.33 & 20.63 & 42.10 & 0.97 & 4.63 & 6.17 & 11.11 & 4.55 & 303.30 \\
\hline 39 & NDML-NS-11 & 5.30 & 14.37 & 42.67 & 46.33 & 54.00 & 20.07 & 40.17 & 0.88 & 5.13 & 6.61 & 13.86 & 4.44 & 295.98 \\
\hline 40 & NDML-NS-12 & 7.90 & 14.03 & 50.70 & 51.23 & 60.33 & 20.87 & 41.80 & 0.97 & 6.70 & 6.33 & 11.89 & 6.15 & 409.98 \\
\hline Gra & d Mean & 7.38 & 10.88 & 46.97 & 49.11 & 58.77 & 30.29 & 31.17 & 0.90 & 4.72 & 5.69 & 11.95 & 4.37 & 376.90 \\
\hline C.V & & 5.83 & 6.38 & 2.97 & 3.08 & 2.09 & 3.30 & 4.02 & 4.45 & 11.16 & 8.81 & 11.59 & 9.27 & \\
\hline S.E. & $m \pm$ & 0.25 & 0.40 & 0.80 & 0.87 & 0.71 & 0.58 & 0.72 & 0.02 & 0.30 & 0.29 & 0.80 & 0.23 & \\
\hline C.D & at $5 \%$ & 0.70 & 1.13 & 2.27 & 2.46 & 1.99 & 1.62 & 2.04 & 0.06 & 0.86 & 0.81 & 2.25 & 0.66 & \\
\hline
\end{tabular}


Table.2 The most desirable genotypes identified for 13 characters in bottle gourd

\begin{tabular}{|c|c|c|c|c|c|c|}
\hline \multirow{2}{*}{$\begin{array}{l}\text { S. } \\
\text { No }\end{array}$} & \multirow[t]{2}{*}{ Characters } & \multicolumn{5}{|c|}{ Genotypes } \\
\hline & & 1 & 2 & 3 & 4 & 5 \\
\hline 1 & Node number to first staminate flower anthesis & NDBG-613-1 & NDBG-619-6-4 & NDBG-619-11-2 & NDBG-619-11-3 & NDBG-701 \\
\hline 2 & Node number to first pistillate flower anthesis & NDBG-613-1 & NDBG-613-2 & NDML-NS-11 & NDML-NS-12 & NDBG-619-6-4 \\
\hline 3 & Days to first staminate flower anthesis & NDML-NS-12 & NDBG-619-6-4 & NDBG-613-1 & NDML-SS-5 & NDML-NS-10 \\
\hline 4 & Days to first pistillate flower anthesis & NDML-SS-5 & NDBG-613-1 & NDBG-619-6-4 & NDBG-613-2 & NDML-SS-1 \\
\hline 5 & Days to first harvest & NDBG-613-2 & NDBG-613-1 & NDML-SS-1 & NDML-NS-10 & NDBG-601-3 \\
\hline 6 & Fruit length/polar length (cm) & NDBG-601-3 & NDBG-619-6-4 & NDBG-604-4 & NDBG-601-2 & NDBG-619-11-2 \\
\hline 7 & Fruit circumference $(\mathrm{cm})$ & NDML-SS-18 & NDML-SS-3 & NDML-NS-10 & NDML-NS-12 & RDPG-NS-1 \\
\hline 8 & Fruit weight (kg) & NDML-SS-18 & NDBG-601-3 & NDML-SS-17 & NDML-NS-10 & NDML-NS-12 \\
\hline 9 & Number of fruits per plant & NDBG-619-11-2 & NDBG-703 & NDBG-701 & NDBG-601-2 & NDBG-619-11-3 \\
\hline 10 & Vine length at the time of harvest (m) & NDBG-601-3 & NDBG-619-11-3 & NDML-SS-5 & NDBG-140 & NDML-SS-18 \\
\hline 11 & $\begin{array}{l}\text { Number of primary branches per vine at the } \\
\text { time of last harvest }\end{array}$ & NDBG-601-3 & NDBG-619-6-3 & NDBG-613-3 & NDBG-140 & NDBG-619-11-3 \\
\hline 12 & Fruit yield (kg/plant) & NDBG-619-11-2 & NDML-SS-9 & NDML-NS-12 & NDBG-701 & NDBG-703 \\
\hline 13 & Fruit yield (q/ha) & NDBG-619-11-2 & NDML-SS-9 & NDML-NS-12 & NDBG-701 & NDBG-703 \\
\hline
\end{tabular}

General mean of this trait for all genotypes was (31.17) fruit weight $(\mathrm{kg})$ NDBG-613-2 (0.74) to NDML-SS-18 (1.03) General mean of this trait for all genotypes was (0.91) number of fruits/plant NDML-SS-17 (1.60) to Narendra Dharidar (8.50) General mean of this trait for all genotypes was (4.72) vine length at the time of last harvest (m) NDMLSS-15 (3.84) to NDBG-601-3 (7.30) General mean of this trait for all genotypes was (5.69) primary branches/vine at the time of last harvest, RDPG-NS-3 (6.66) to NDBG-601-3 (15.33) General mean of this trait for all genotypes was (11.95) fruit yield ( $\mathrm{kg} / \mathrm{plant})$ NDBG-613-2 (1.46) to NDBG-619-11-2 (7.95) General mean of this trait for all genotypes was (4.37) fruit yield (q/ha). NDBG-613-2 (97.32) to NDBG-619-11-2 (529.92) General mean of this trait for all genotypes was (376.90) for this character and out of forty genotypes were non-significant.

In the present study to evaluate the germplasm collection the mean of forty genotypes with two checks for 13 characters is presented in Table 1. Very wide ranges of variation in mean performance of genotypes were observed for all the characters under study. The comparison of mean performance of forty genotypes with two checks for 13 traits, revealed existence of very high level of variability in the germplasm collection. The genotypes showing very high mean performance in desirable direction for various characters are listed in Table 2, which may also be used for donors for improving the characters for which they had high mean performance.

\section{Acknowledgement}

The senior author are thankful to Prof. Sheo Pujan Singh for providing facilities Department of Vegetable Science, N.D.U.A.\&T. Kumarganj, Faizabad (U.P.) for providing necessary facilities.

\section{References}

Anonymous (2004). FAO website: www.fao.org

Dubey, (2012). Evaluation of morphologically distinct groups of bottle gourd genotypes under fusarium wilt sick plot condition. Ph.D., thesis submitted to N.D. Univ. of Agri. \& Techn., Kumarganj, Faizabad. (U.P.).

Dwivedi, A. (2000). Documentation of germplasm and genetic studies in bottle gourd. (Lagenaria siceraria (Molina) StandL). M.Sc. (Ag.) Thesis, Department of Vegetable Science, N.D. 
Dniv. of Agril. \& Tech. Kumarganj, Faizabad, (U.P.).

Hawlader, M.S.H.; Haque, M.M. and Islam, M.S. (1999). Variability, correlation and path analysis in bottle gourd. Bangladesh J. of Scientific and Indust. Res., 34: 50-54.

Singh, D.K. and Kumar, R. (2002). Studies on the genetic variability in bottle gourd. Prog. Hort., 34: 99-101.

Singh, S. P. (2012). Final Progress Report of the project entitled 'Off season cultivation of cucurbits in eastern Uttar Pradesh' Department of Vegetable Science, N.D. Univ. of Agri. \& Techn., Kumarganj, Faizabad, (U.P.).

Singh, S. P. (2013). Cucurbits: Biodiversity, Breeding and production in Uttar
Pradesh, Published by Uttar Pradesh state Biodiversity Board, Lucknow (U.P.) $108 \mathrm{p}$.

Singh, S.P.; Singh, N.K. and Maurya, I.B (1996b). Genetic variability and correlation studies in bottle gourd (Lagenaria siceraria (Mol.) Standl.). P.K.V.J., 20: 88-89.

Tyagi, L.D. (1972). Variability and correlation studies in bottle gourd (Lagenaria siceraria (Mol.) Standl.). Indian J. Hort., 29: 219-222.

Yadav, J.R.; Yadav, A.; Srivastava, J.P.; Mishra, G.; Parihar, N.S. and Singh, P.B. (2008). Study on variability heritability and genetic advance in bottle gourd [Lagenaria siceraria (Molina) Standl.]. Prog Res., 3: 70-72.

\section{How to cite this article:}

Akshay Jain, S.P. Singh, Rajneesh Shukla and Sriom. 2018. Evaluation on Mean Performance in Bottle Gourd [Lagenaria siceraria (Molina) Standl] Genotypes. Int.J.Curr.Microbiol.App.Sci. 7(04): 2239-2243. doi: https://doi.org/10.20546/ijcmas.2018.704.255 